\title{
First report of natural Wolbachia infection in wild Anopheles funestus population in Senegal
}

\author{
El Hadji Amadou Niang ${ }^{1,2,3^{*}}$ (D) Hubert Bassene ${ }^{3,4}$, Patrick Makoundou ${ }^{5}$, Florence Fenollar ${ }^{4}$, Mylène Weill ${ }^{5}$ \\ and Oleg Mediannikov ${ }^{1}$
}

\begin{abstract}
Background: Until very recently, Anopheles were considered naturally unable to host Wolbachia, an intracellular bacterium regarded as a potential biological control tool. Their detection in field populations of Anopheles gambiae sensu lato, suggests that they may also be present in many more anopheline species than previously thought.

Results: Here, is reported the first discovery of natural Wolbachia infections in Anopheles funestus populations from Senegal, the second main malaria vector in Africa. Molecular phylogeny analysis based on the $16 \mathrm{~S}$ rRNA gene revealed at least two Wolbachia genotypes which were named $W A n f u-A$ and $W A n f u-B$, according to their close relatedness to the $A$ and $B$ supergroups. Furthermore, both wAnfu genotypes displayed high proximity with wAnga sequences previously described from the An. gambiae complex, with only few nucleotide differences. However, the low prevalence of infection, together with the difficulties encountered for detection, whatever method used, highlights the need to develop an effective and sensitive Wolbachia screening method dedicated to anopheline.

Conclusions: The discovery of natural Wolbachia infection in An. funestus, another major malaria vector, may overcome the main limitation of using a Wolbachia-based approach to control malaria through population suppression and/or replacement.
\end{abstract}

Keywords: Wolbachia, Plasmodium, An. funestus, wAnfu, Malaria, Biological control Senegal

\section{Background}

Wolbachia are intracellular bacteria found in the cytoplasmic vacuoles of different cells of a wide range of invertebrates, including multiple insect species [1]. The success of Wolbachia spp. becoming the most widespread intracellular bacterium, originates from their ability to manipulate the biology of their host to facilitate their maternal transmission to offspring $[2,3]$. The most common of these host reproductive manipulation phenotypes, known as cytoplasmic incompatibility (CI), is considered as a potential biological control alternative or complement to traditional vector control measures [4].

\footnotetext{
*Correspondence: eaniang1@yahoo.fr

${ }^{1}$ Aix Marseille Univ, IRD, AP-HM, MEPHI, IHU-Méditerranée Infection, Marseille, France

Full list of author information is available at the end of the article
}

Over the past decade, two research groups have reported that Wolbachia infection protects against viral RNA infections in Drosophila melanogaster [5, 6]. Subsequently, the successful trans-infection of Aedes aegypti with a Drosophila Wolbachia strain has opened a new era for environmental-friendly control strategies of main mosquitoes-borne diseases using Wolbachia-based strategies [7]. Moreover, successful releases of Wolbachia-infected Aedes aegypti in Australia has provided experimental validation of previous theoretical models of Wolbachia population dynamics and demonstrated the viability of Wolbachia-based vector control strategies [8]. For decades, anopheline mosquitoes that transmit human malaria have been considered resistant or less susceptible to Wolbachia infections due to the failure to detect native Wolbachia infection in 38 species of anopheles $[9,10]$, and the impossibility to obtain stable Wolbachia 
trans-infected anopheline lines [11, 12]. Although, Wolbachia trans-infections have been attempted with success in both Anopheles gambiae [11, 13] and Anopheles stephensi [14], respectively major vectors of human malaria in Africa and the Middle East, and South Asia [15, 16], their impact on Plasmodium development were not always conclusive. The dogma of the absence of native Wolbachia infection in anopheline mosquito has recently changed with the first report of Wolbachia in Anopheles gambiae sensu lato (s.l.) from Burkina Faso [17, 18]; and more recently from Mali [10].

Recent advances in the molecular biology area, including sequencing of $16 \mathrm{~S}$ rRNA, have revolutionized the characterization of several fastidious microorganisms. This is particularly true for intracellular bacteria of the members of the Wolbachia genus [19]. Indeed, phylogenetic analyses of the 16S rRNA gene have revealed that Wolbachia pipientis, the nomen species of the genus, forms a monophyletic clade within the Alphaproteobacteria class, closely related to the Anaplasma, Ehrlichia and Neorickettsia genera of the Anaplasmataceae family [20]. Further phylogenetic analysis based on the 16S rRNA of newly discovered anopheline Wolbachia strains revealed that they belong to a new phylogenetic group called wAnga related to, but distinct from Wolbachia infecting other arthropods [9, 10, 17]. Baldini et al. [17], explain previous failure of detecting Wolbachia in anopheline mosquitoes as possibly due to the lack of sufficiently sensitive detection systems and developed a nested amplification method. In addition, the new wAnga genotypes display a low degree of sequence conservation compared to previously described genotypes isolated in other insects leading to unsuccessful amplification of several genes commonly used for Multi-Locus Sequence Typing (MLST) Wolbachia universal genotyping tool [17]. These discoveries suggest that more anopheline species, including others major malaria vectors, may also be infected by Wollbachia.

In this study, is reported the first discovery of natural Wolbachia infections in Anopheles funestus in Senegal, the second main malaria vector in Africa.

\section{Methods}

\section{Study area and mosquito collection}

A total of 247 adult females of An. funestus collected during the raining season of the year 2014 were screened. Samples were randomly selected from an existing mosquito collection from the longitudinal cohort study conducted since 1990 in the village of Dielmo [Senegal]. Detail of the study village and mosquito collection methods are described elsewhere [21, 22]. All specimens were identified morphologically as An. funestus using the taxonomic key of Gillies and Meillon [23] prior to molecular detection and genotyping of Wolbachia spp.

\section{Molecular detection and phylogenetic genotyping of Wolbachia}

Genomic DNA was extracted from the abdomen of individual mosquito using the Biorobot EZ1 System with the EZ1 DNA tissue kit [Qiagen, Court a boeuf, France] following the manufacturer's instructions. Individual mosquitoes were screened as described in Shaw et al. [18] using both the standard [W16S-Spec] [24] and the nested PCR [W16S-Nested] [17] protocols. A third qPCR assay [W16S-qPCR] recently developed [10] was used to confirm Wolbachia infection in An. funestus.

The nested-16S rDNA Wolbachia primers were used to generate a 340-412-bp fragment according to Baldini et al. [17]. The PCR products of all Wolbachia-positive samples were purified by filtration using NucleoFast ${ }^{\circledR}$ 96 PCR DNA purification plate then amplified using the BigDye $^{\mathrm{TM}}$ Terminator v3.1 Cycle Sequencing Kit [Applied Biosystems, Foster City, CA]. The BigDye PCR products were purified on the Sephadex G-50 Superfine gel filtration resin prior the sequencing on the ABI Prism 3130XL.

\section{Phylogenetic analysis of Wolbachia}

Nucleotide sequences were edited using ChromasPro 2.0.0, then aligned against close reference sequences of $w M e l$ [LC108848] and wAlb, [AM999887], respective representatives of the A and B supergroups; and three wAnga genotypes, previously described in field populations of An. gambiae s.l. from Burkina [KJ728749 and KJ728744] and from Mali [MF944114]. All the reference sequences were retrieved from the GenBank database and the alignment was performed using the ClustalW application within Bioedit v.7.2.5. [25]. Nucleotides conservation between the An. funestus Wolbachia sequences comparatively to reference sequences was visualized on CLC Sequence Viewer 7 (CLC Bio Qiagen, Aarhus, Denmark).

The maximum likelihood phylogenetic tree was inferred on Topali v2.5 [26], based on the Kimura threesubstitution-type substitution model [27].

\section{Results}

Wolbachia infection in wild Anopheles funestus populations from Senegal

Wolbachia DNA was detected in three specimens out of 247 females of An. funestus tested, which corresponds to a frequency of infection of $1.21 \%$. This is the first report of Wolbachia infection among natural population of $A n$. funestus in Senegal. Despite several attempt, the quantitative PCR developed by Gomez et al. [10] failed to 
amplify the three positive samples amplified with the nested PCR.

\section{Phylogenetic analysis of Wolbachia infecting Anopheles funestus}

Phylogenetic analysis of the $16 \mathrm{~S}$ rRNA gene revealed that the An. funestus samples from Senegal infected with at least two Wolbachia genotypes which cluster with the A or B clades (Figs. 1, 2), and were named wAnfu-A and $w A n f u$-B. Further sequences comparisons showed the identity of wAnfu-A to $w M e l$ and $w A n g a-\mathrm{BF}$ VK7; and the close relatedness of $w A n f u-B$ sequence to $w A l b$ and wAnga-BF VK5 (Fig. 2). Noteworthy, despite their proximity, slight levels of divergence were found between the $w A n f u$ genotypes of An. funestus and wAnga strains previously found in the An. gambiae complex. Furthermore, mutations differentiating the two $w A n f u$ variants suggest further diversification within Wolbachia strain infecting An. funestus.

\section{Discussion}

This is the first report of Wolbachia infection among natural population of An. funestus in Senegal, the second main malaria vector in the African continent [15]. This extends the presence of Wolbachia to another anopheline species since its first discovery in field populations of $A n$. gambiae, Anopheles coluzzii and Anopheles arabiensis from Burkina Faso $[17,18]$ and in An. gambiae and An. coluzzii from Mali [10]. However, the prevalence of Wolbachia in An. funestus is lower compared to the previous reports, with 46\% (275/602) in 2014 in Burkina Faso and varying spatially and temporally in Mali with a minimal infection rate of 45\% in Dangassa in 2015 reaching 95\% $(38 / 40)$ in Kenieroba in 2016. Such differences in prevalence may be explained by lower infection rates in $A n$. funestus compared to An. gambiae species [10, 17, 18] due to biological, immunological or any other factors. Another hypothesis could be a weaker infection density in An. funestus [quantity of Wolbachia per cell] that could prevent its correct detection. The failure of the $\mathrm{qPCR}$ assay developed by Gomez et al. [10] to amplify the three positive samples, confirmed independently in two laboratories (in Marseille and Montpellier), suggests such an explanation, since the sequences of the primers designed for this qPCR are conserved in the Wolbachia of An. funestus. Thus, the wAnfu prevalence detected in Senegal is minimal and could be higher with a more efficient detection system. Moreover, a certain level of divergence

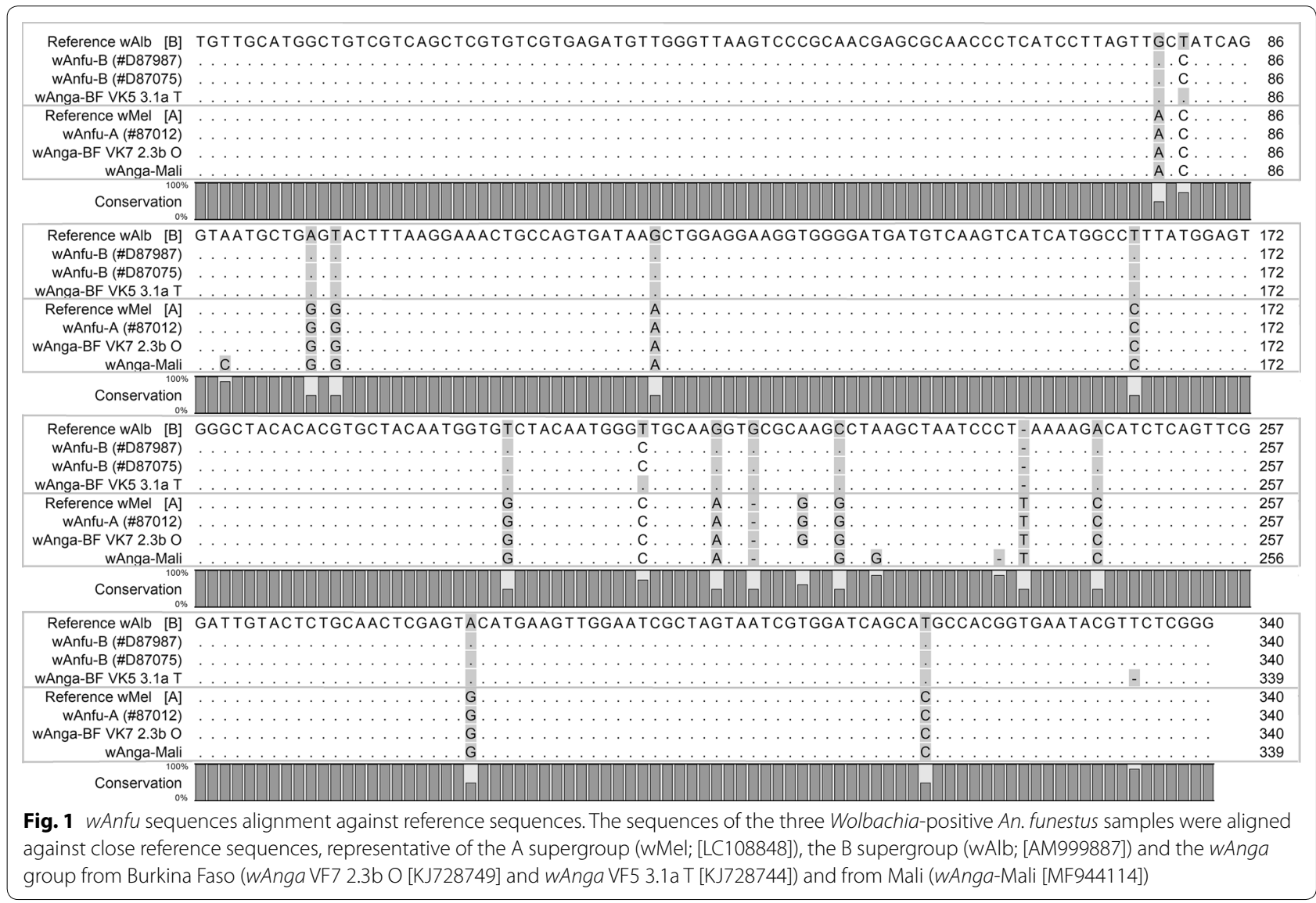




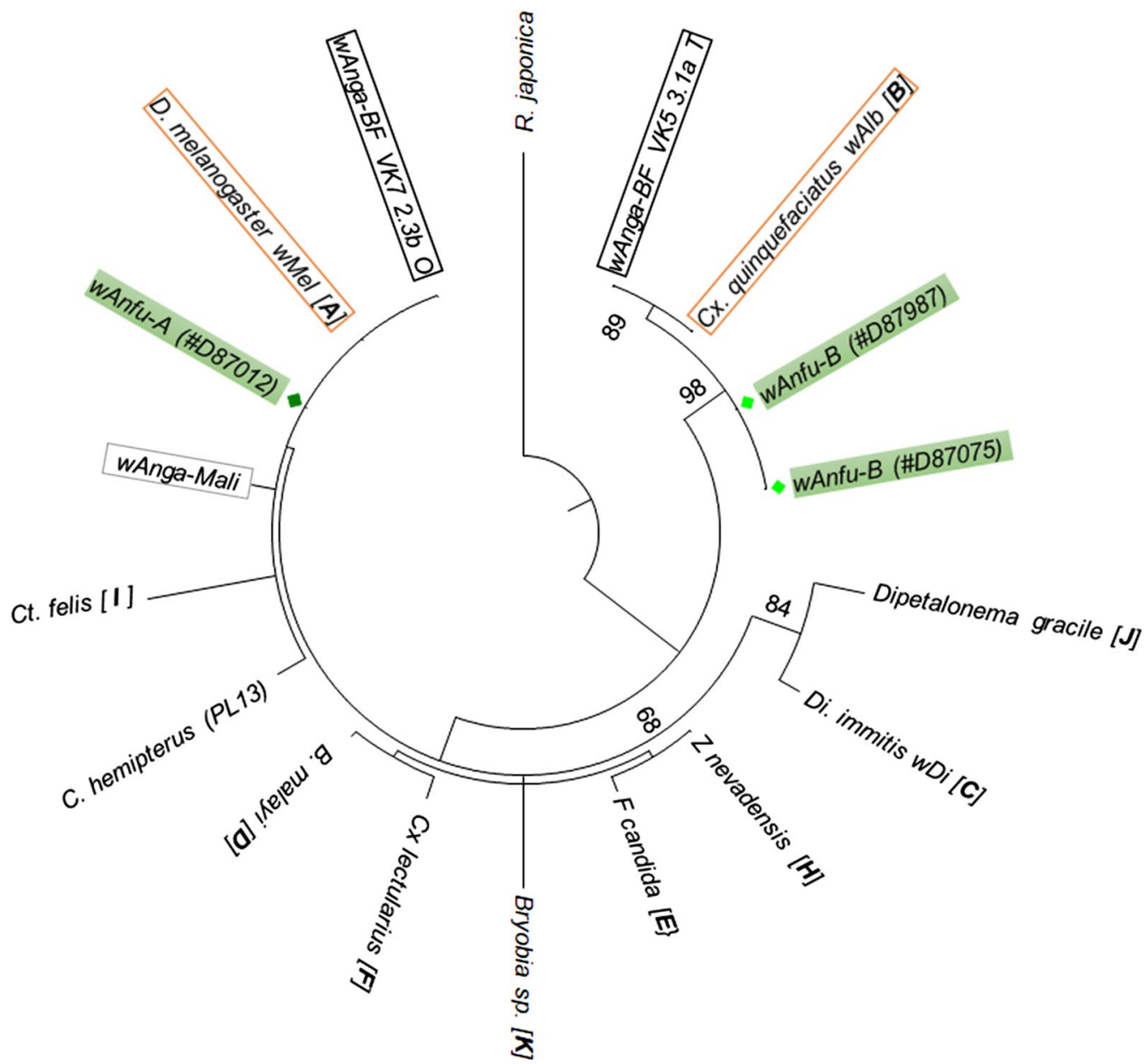

Fig. 2 Molecular Phylogenetic analysis by the Maximum Likelihood method. The evolutionary history based on the 340 bp sequence of the partial Wolbachia 165 rRNA gene was inferred using the Maximum Likelihood method based on the Kimura three-substitution-type model [27]. Wolbachia genotypes of An. funestus are highlighted by green shaded boxes. The two red boxes show the A and B supergroups reference sequences. The wAnga genotypes from Burkina Faso are highlighted with black boxes and wAnga-Mali is illustrated by a grey box. The different Wolbachia supergroups are mentioned in brackets after each reference genotype name

between $w A n f u$ and $w A n g a$ sequences previously found in the An. gambiae complex [10,17, 18] could also prevent efficient amplification and thus detection. Indeed, Baldini et al. [17], also attributed the previous failure to detect Wolbachia infection in wild anopheline populations by non-optimal detection tools, due to the genetic divergence of the new wAnga strain from Wolbachia found in other insects. Finally, because of the low prevalence (1.2\%) of Wolbachia infection in An. funestus, probably due to the low infection level that can lead to qPCR failures, and in the absence of vertical transmission history, we cannot exclude the possibility of an occasional unstable infection.

Phylogenetic analysis showed that natural populations of Senegalese An. funestus harbour at least two distinct
Wolbachia genotypes; clustering respectively with the clade A and B commonly encountered in the Arthropoda phylum [3]. The new genotypes were therefore named $w A n f u-A$ and $w A n f u-B$, according to their respective relatedness to the A and B supergroups. Indeed, further analyses revealed the similarity of $w A n f u$-A to $w M e l$ and $w A n g a-\mathrm{BF}$ VK7; while $w A n f u-\mathrm{B}$ was closer to $w A l b$ and wAnga-BF VK5. However, multi-sequence alignment revealed that despite their proximity, the An. funestus Wolbachia genotypes were slightly different from the previously described wAnga infecting several species of the An. gambiae complex [17, 18].

Moreover, the appearance of some mutations also favours diversification between Wolbachia in An. funestus, with at least two variants. Given the suspected 
further divergence between anopheline Wolbachia groups, some genotypes may have been missed. It is, therefore, critical to vary detection methods targeting more genes. A classic approach to characterize Wolbachia genotypes and clades is based on the MLST system using internal fragments of five ubiquitous genes (gatB, coxA, hcpA, fbpA, and ftsZ) [28]. However, as shown by Baldini et al. [17], their newly identified wAnga genotype was highly divergent from groups isolated in other insects, leading potentially to the presence of null alleles if mutations have occurred in the region targeted to design the standard primers of the MLST universal genotyping tool. This seems to be corroborated by Gomes et al. [10], who failed to successfully amplify the gat $B$ and fts $Z$ genes. There is, therefore, an urgent need to develop an optimal screening method, but also a specific MLST system for anopheline Wolbachia. A critical and challenging step for this would be the isolation and whole genome sequencing of, as much as possible, Wolbachia genotypes infecting anopheline mosquitoes to come-up with a specific MLST system and potentially a more efficient screening system. Then, more data on Wolbachia prevalence will be required to further assess their potential role in impeding the development of Plasmodium or any other parasites.

This is the first report of the presence of Wolbachia spp. in An. funestus from Senegal. Stable natural Wolbachia carriage among main malaria vectors may overcome the main limitation of using a Wolbachia-based approach to control malaria through population suppression and/or replacement. However, further studies are needed to better characterize the diverse Wolbachia groups infecting anopheline mosquitoes prior they could be efficiently used as control tool.

\section{Abbreviations \\ DNA: deoxyribonucleic acid; MLST: multilocus sequence typing; PCR: poly- merase chain reaction; qPCR: quantitative polymerase chain reaction; RNA: ribonucleic acid.}

\section{Authors' contributions}

EAN, FF, MW and OM designed research; EAN, HB and PM performed the field collection and the lab work. All the authors have drafted and reviewed the manuscript. All authors read and approved the final manuscript.

\footnotetext{
Author details

1 Aix Marseille Univ, IRD, AP-HM, MEPHI, IHU-Méditerranée Infection, Marseille, France. ${ }^{2}$ Laboratoire d'Ecologie Vectorielle et Parasitaire, Faculté des Sciences et Techniques, Université Cheikh Anta Diop, Dakar, Senegal. ${ }^{3}$ VITROME, Campus International, UCAD-IRD, Dakar, Senegal. ${ }^{4}$ Aix Marseille Univ, IRD, AP-HM, SSA, VITROME, IHU-Méditerranée Infection, Marseille, France. ${ }^{5}$ Institut des Sciences de I'Evolution (ISEM), CNRS-Université de Montpellier-IRD-EPHE, Montpellier, France.
}

\section{Acknowledgements}

The authors gratefully acknowledge the field team and the population of Dielmo.

\section{Competing interests}

The authors declare that they have no competing interests.

\section{Availability of data and materials}

The datasets used and/or analysed during the current study are available from the corresponding author on reasonable request.

\section{Consent for publication}

Not applicable.

\section{Ethics approval and consent to participate}

Not applicable.

\section{Funding}

This work was supported by IHU Méditerranée Infection, Marseille, France, the French Government under the «Investissements d'avenir» (Investments for the Future) program managed by the Agence Nationale de la Recherche (ANR, fr: National Agency for Research), (reference: Méditerranée Infection 10-IAHU03), and by the Région Provence Alpes Côte d'Azur and European funding FEDER PRIMI.

\section{Publisher's Note}

Springer Nature remains neutral with regard to jurisdictional claims in published maps and institutional affiliations.

Received: 21 June 2018 Accepted: 30 October 2018

Published online: 06 November 2018

\section{References}

1. Ilinsky Y, Kosterin OE. Molecular diversity of Wolbachia in Lepidoptera: prevalent allelic content and high recombination of MLST genes. Mol Phylogenet Evol. 2017;109:164-79.

2. Werren JH, Baldo L, Clark ME. Wolbachia: master manipulators of invertebrate biology. Nat Rev Microbiol. 2008;6:741-51.

3. Glowska E, Dragun-Damian A, Dabert M, Gerth M. New Wolbachia supergroups detected in quill mites (Acari: Syringophilidae). Infect Genet Evol. 2015;30:140-6.

4. Brelsfoard CL, Dobson SL. Wolbachia-based strategies to control insect pests and disease vectors. As Pac J Mol Biol Biotechnol. 2009;17:55-63.

5. Hedges LM, Brownlie JC, O'Neill SL, Johnson KN. Wolbachia and virus protection in insects. Science. 2008;322:702.

6. Teixeira L, Ferreira A, Ashburner M. The bacterial symbiont Wolbachia induces resistance to RNA viral infections in Drosophila melanogaster. PLoS Biol. 2008:6:e1000002.

7. Moreira LA, Iturbe-Ormaetxe I, Jeffery JA, Lu G, Pyke AT, Hedges LM, et al. A Wolbachia symbiont in Aedes aegypti limits infection with Dengue, Chikungunya, and Plasmodium. Cell. 2009;139:1268-78.

8. Jiggins FM, Smidler A, Catteruccia F, Snoeck K, Day J, Jiggins F. The spread of Wolbachia through mosquito populations. PLoS Biol. 2017;15:e2002780.

9. Joshi D, Pan X, McFadden MJ, Bevins D, Liang X, Lu P, et al. The maternally inheritable Wolbachia wAlbB induces refractoriness to Plasmodium berghei in Anopheles stephensi. Front Microbiol. 2017:8:1-11.

10. Gomes FM, Hixson BL, Tyner MDW, Ramirez JL, Canepa GE, Alves e Silva $T L$, et al. Effect of naturally occurring Wolbachia in Anopheles gambiae s.l. mosquitoes from Mali on Plasmodium falciparum malaria transmission. Proc Natl Acad Sci USA. 2017;114:12566-71.

11. Kambris Z, Blagborough AM, Pinto SB, Blagrove MSC, Godfray HCJ, Sinden RE, et al. Wolbachia stimulates immune gene expression and inhibits Plasmodium development in Anopheles gambiae. PLoS Pathog. 2010;6:e1001143.

12. Murdock CC, Blanford S, Hughes GL, Rasgon JL, Thomas MB. Temperature alters Plasmodium blocking by Wolbachia. Sci Rep. 2014;4:3932.

13. Hughes $G L$, Koga R, Xue P, Fukatsu T, Rasgon JL. Wolbachia infections are virulent and inhibit the human malaria parasite Plasmodium falciparum in Anopheles gambiae. PLoS Pathog. 2011;7:e1002043. 
14. Bian G, Joshi D, Dong Y, Lu P, Zhou G, Pan X, et al. Wolbachia invades Anopheles stephensi populations and induces refractoriness to Plasmodium infection. Science. 2013;340:748-50.

15. Sinka ME, Bangs MJ, Manguin S, Coetzee M, Mbogo CM, Hemingway J, et al. The dominant Anopheles vectors of human malaria in Africa, Europe and the Middle East: occurrence data, distribution maps and bionomic precis. Parasit Vectors. 2010;3:117.

16. Sinka ME, Bangs MJ, Manguin S, Rubio-Palis Y, Chareonviriyaphap T, Coetzee $M$, et al. A global map of dominant malaria vectors. Parasit Vectors. 2012;5:69.

17. Baldini F, Segata N, Pompon J, Marcenac P, Robert Shaw W, Dabiré RK, et al. Evidence of natural Wolbachia infections in field populations of Anopheles gambiae. Nat Commun. 2014;5:3985.

18. Shaw WR, Marcenac P, Childs LM, Buckee CO, Baldini F, Sawadogo SP, et al. Wolbachia infections in natural Anopheles populations affect egg laying and negatively correlate with Plasmodium development. Nat Commun. 2016;7:11772.

19. Werren $J \mathrm{H}, \mathrm{O}^{\prime} \mathrm{N}$ eill SL. The evolution of heritable symbionts. In: O'Neill SL, Hoffmann AA, Werren JH, editors. Influential passengers: inherited microorganisms and invertebrate reproduction. Oxford: Oxford University Press; 1997.

20. La Scola B, Bandi C, Raoult D. Wolbachia. Bergey's Manual of Systematics of Archaea and Bacteria. Chichester: Wiley; 2015

21. Trape JF, Tall A, Diagne N, Ndiath O, Ly AB, Faye J, et al. Malaria morbidity and pyrethroid resistance after the introduction of insecticide-treated bednets and artemisinin-based combination therapies: a longitudinal study. Lancet Infect Dis. 2011;11:925-32.

22. Trape JF, Tall A, Sokhna C, Ly AB, Diagne N, Ndiath O, et al. The rise and fal of malaria in a west African rural community, Dielmo, Senegal, from 1990 to 2012: a 22 years longitudinal study. Lancet Infect Dis. 2014;14:476-88.

23. Gillies MT, De Meillon B. The Anophelinae of Africa south of the Sahara (Ethiopian Zoogeographical Region). Johannesburg: South African Institute for Medical Research; 1968.

24. Werren $\mathrm{JH}$, Windsor DM. Wolbachia infection frequencies in insects: evidence of a global equilibrium? Proc R Soc Lond B. 2000;267:1277-85.

25. Hall TA. BioEdit: a user-friendly biological sequence alignment editor and analysis program for Windows 95/98/NT. Vol. 41, Nucleic Acids Symposium Series. 1999. p. 95-8.

26. Milne I, Lindner D, Bayer M, Husmeier D, McGuire G, Marshall DF, et al. TOPALi v2: a rich graphical interface for evolutionary analyses of multiple alignments on HPC clusters and multi-core desktops. Bioinformatics. 2009;25:126-7.

27. Kimura M. Estimation of evolutionary distances between homologous nucleotide sequences (molecular evolution/comparison ofbase sequences/base substitution rate/neutral mutation-random drift hypothesis). Genetics. 1981;78:454-8.

28. Baldo L, Hotopp JCD, Jolley KA, Bordenstein SR, Biber SA, Choudhury $\mathrm{RR}$, et al. Multilocus sequence typing system for the endosymbiont Wolbachia pipientis. Appl Environ Microbiol. 2006;72:7098-110.
Ready to submit your research? Choose BMC and benefit from:

- fast, convenient online submission

- thorough peer review by experienced researchers in your field

- rapid publication on acceptance

- support for research data, including large and complex data types

- gold Open Access which fosters wider collaboration and increased citations

- maximum visibility for your research: over 100M website views per year

At BMC, research is always in progress.

Learn more biomedcentral.com/submissions 